\title{
Effects Of Exercise Training With Dietary Restriction On Arterial Stiffness, Central Hemodynamic Parameters And Cardiac Autonomic Function In Obese Adolescents
}

This article was published in the following Dove Press journal:

Diabetes, Metabolic Syndrome and Obesity: Targets and Therapy

\author{
Junhao Huang' \\ Qinhao Lai' \\ Dan Wang' \\ Honggang Yin' \\ Jingwen Liao' \\ Shen Wang ${ }^{I-3}$ \\ Fengpeng $\mathrm{Xu}^{\prime}$ \\ Xiaohui Hou ${ }^{2}$ \\ Min $\mathrm{Hu}^{\prime}$
}

'Guangdong Provincial Key Laboratory of Sports and Health Promotion, Scientific Research Center, Guangzhou Sport University, Guangzhou, Guangdong, People's Republic of China; ${ }^{2}$ Department of Sports and Health, Guangzhou Sport University, Guangzhou, Guangdong, People's Republic of China; ${ }^{3}$ School of Kinesiology, Shanghai University of Sport, Shanghai, People's Republic of China

Correspondence: Min Hu

Scientific Research Center, Guangzhou Sport University, 1268 Middle Guangzhou Avenue, Guangzhou 510500, People's

Republic of China

Tel/fax +86-20-38027669

Email whoomin@aliyun.com

Xiaohui Hou

Department of Sports and Health, Guangzhou Sport University, 1268 Middle Guangzhou Avenue, Guangzhou 510500,

People's Republic of China

Tel +86-20-38025087

Fax +86-20-38027669

Email lilyhxh@163.com

\begin{abstract}
Purpose: This study aimed to assess the effects of exercise with dietary restriction on arterial stiffness, central hemodynamics, and cardiac autonomic function in obese adolescents.

Patients and methods: Twenty-one obese adolescents completed a 6-week exercise and dietary program. Body composition and metabolic parameters were measured. Carotidfemoral pulse wave velocity (cfPWV) and brachial-ankle pulse wave velocity (baPWV) were used to assess central and systemic arterial stiffness, respectively. Using applanation tonometry, the analyses of central hemodynamic parameters [central blood pressure, heart rate (HR), augmentation index normalized at $75 \mathrm{bpm}$ (AIx75), and subendocardial viability ratio (SEVR)] were performed. To determine cardiac autonomic function, heart rate variability (HRV) was analyzed by standard deviation of normal R-R intervals (SDNN), root mean square of successive $\mathrm{R}-\mathrm{R}$ interval differences (RMSSD), percentage of successive $\mathrm{R}-\mathrm{R}$ intervals that differed by $>50 \mathrm{~ms}$ (pNN50), total power (TP), low-frequency (LF) and highfrequency (HF) power, and LF/HF ratio.

Results: Following the intervention, obese adolescents had reductions in body mass index, body fat percentage, brachial systolic and diastolic blood pressure, and HR; and they had shown improvements in lipid profiles. There were reductions in both cfPWV and baPWV following the intervention. Moreover, there were reductions in AIx75, aortic systolic and diastolic blood pressure and an augmentation in SEVR after intervention. The intervention increased cardiac autonomic function (determined by increased SDNN, RMSSD, pNN50, $\mathrm{HF}$, and reduced LF/HF). Furthermore, we observed a correlation between increased cardiac autonomic function (SDNN, RMSSD, pNN50 and HF) and increased central hemodynamics, as measured by SEVR and AIx75.
\end{abstract}

Conclusion: Obese adolescents had increased central hemodynamics and autonomic function with reduced arterial stiffness after exercise and dietary restriction. Moreover, a positive correlation between an enhancement of cardiac autonomic function and an augmentation in central hemodynamics was found after the intervention.

Keywords: obesity, adolescents, exercise, cardiac autonomic function, arterial stiffness, diet

\section{Introduction}

The prevalence of obesity among children and adolescents is rapidly increasing in modern societies. ${ }^{1}$ In 2016, more than 340 million children and adolescents (aged between 5 and 19 years) were reported as being overweight or obese. ${ }^{2}$ Importantly, childhood and adolescent obesity are associated with an increased risk for 
developing type 2 diabetes mellitus and atherosclerotic cardiovascular disease (CVD) in adulthood. ${ }^{3}$

Abnormalities in arterial and cardiac function play a critical role in the increased risk of cardiovascular events in the obese. ${ }^{2-4}$ Obesity-induced atherosclerosis can be characterized by arterial stiffness. ${ }^{7}$ Aortic arterial stiffness could be evaluated by carotid-femoral pulse wave velocity (cfPWV), the gold standard for noninvasive assessment of arterial stiffness. ${ }^{8}$ In comparison of cfPWV, brachial-ankle PWV (baPWV) is determined partly by systemic arterial stiffness. Greater cfPWV and baPWV have been reported in children and adolescents with obesity. ${ }^{9,10}$

Using applanation tonometry, the analysis of central hemodynamic parameters [central blood pressure, heart rate (HR), augmentation index (AIx), AIx normalized at 75 bpm (AIx75), and subendocardial viability ratio (SEVR)] is performed, which can be used to assess the central blood pressure, aortic vascular function, cardiac blood supply and endocardial perfusion. Impaired central hemodynamics are independent risk factors for CVD. ${ }^{11}$

Autonomic activity assessment is a potentially useful clinical tool in early identification of children and adolescents at CVD risk. ${ }^{3}$ Cardiac autonomic function could be evaluated by heart rate variability (HRV). Furthermore, studies have reported that lower HRV is shown in obese children and adolescents, associated with impaired arterial and cardiac function. ${ }^{12-14}$

Previous study has indicated that a high calorie intake or sedentary lifestyle dramatically increases the incidence of obesity. ${ }^{5}$ Generally, physical activity and diet intervention are beneficial for glucose metabolism, skeletal muscle function, bone stability, psychological well-being, and other organ functions. ${ }^{4}$ However, in the current study, we entirely focus on the effects of combined exercise and diet intervention on cardiac and vascular function including PWV, central hemodynamic parameters, and HRV. We hypothesized that obese adolescents would exhibit augmented central hemodynamics and increased autonomic function with reduced arterial stiffness following a 6-week of exercise and diet intervention.

\section{Materials And Methods}

\section{Participants}

Obese participants aged 10-16 years were recruited from a weight loss camp between July and August 2017. Participants who satisfied the obesity diagnosis criteria [body mass index (BMI) equal to or greater than the 95th percentile for age and sex] were included. ${ }^{16}$ All participants were free of metabolic, gastrointestinal, or cardiac disease. None of the participants were taking medications which affected energy expenditure or were losing weight in the past three months whether by diet, taking weight-loss medication or more physical exercise than normal. All participants and their parents signed written informed consent prior to participation. This study was approved by the Ethics Committee of Guangzhou Sport University and conducted in accordance with the Declaration of Helsinki. The trial was registered in ClinicalTrials.gov (NCT03762629). Of the thirty enrolled participants, twenty-three met the criteria for inclusion and were selected for the training program. Twenty-one participants $(\mathrm{M} / \mathrm{F}, 11 / 10)$ who completed the program and provided valid measurements at both pre- and post-intervention were included in the analysis (TREND flow diagram, Figure 1).

\section{Exercise And Diet Intervention}

The full details of exercise and diet intervention have been published in our previous study. ${ }^{17}$ In brief, participants were provided with a calorie-restricted but nutritionally complete diet based on their age. The amount of diet provided met the basal metabolic rate requirement calculated using the Mifflin equation, ${ }^{6}$ recommended to determine resting energy expenditure in overweight and obese persons. ${ }^{7}$ Dietary plans were made individually according to their own basal metabolic rate. The diet was provided with $60 \%$ carbohydrate, $20 \%$ protein, and $20 \%$ fat, and energy intake was divided as $30 \%$ of total daily calories at breakfast, $40 \%$ at lunch, and $30 \%$ at dinner. The dietitians and nutritionists prepared and supervised all meals. Participants

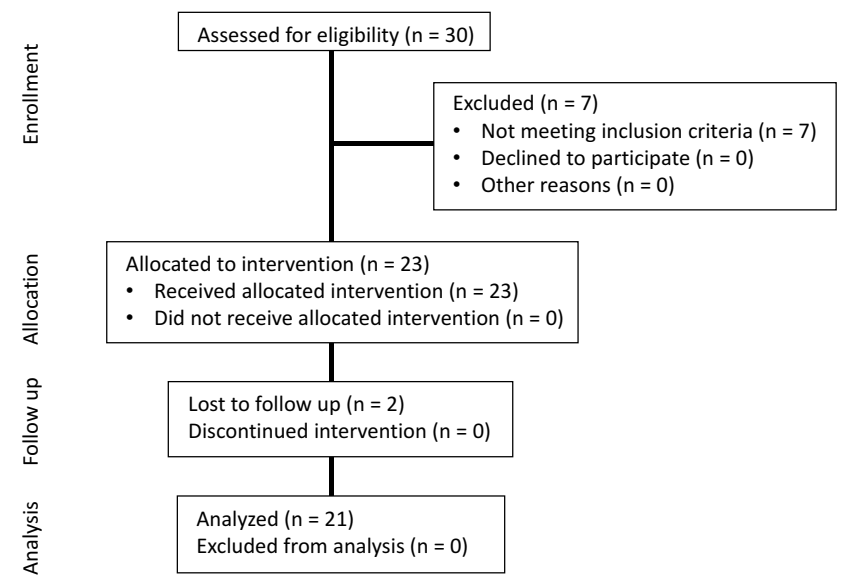

Figure I Flow diagram of participants through the study. 
performed an exercise training program 6 days/week for 6 weeks. The program was primarily comprised of various types of aerobic exercises such as bicycling, walking, running, dancing, and ball games for $5 \mathrm{hrs} /$ day. It was also supplemented by strength training. The endurance exercises involved moderate (70-85\% of maximum heart rate) and high intensity ( $\sim 90 \%$ of maximum heart rate) training. Strength training was conducted 2-3 times per week at $40-50 \%$ maximal strength for $2-3$ sets of $12-15$ repetition maximum with 2-3 mins of rest between sets.

\section{Body Composition And Metabolic Parameters}

Height and weight were determined and BMI was calculated $\left(\mathrm{kg} / \mathrm{m}^{2}\right)$. Body composition was assessed by a body composition analyzer (Inbody 370, Biospace, Seoul, Korea). Fasting blood samples were collected for the assessment of the metabolic parameters [total cholesterol, triglycerides, high-density lipoprotein cholesterol (HDL-c), low-density lipoprotein cholesterol (LDL-c), and glucose].

\section{Arterial Stiffness And Hemodynamic Measurements}

The details for arterial stiffness and hemodynamic measurements were previously described. ${ }^{8}$ In brief, brachial systolic/ diastolic blood pressure (SBP/DBP) were measured using a sphygmomanometer (Omron, 705 IT, Omron Health Care, Japan). Arterial stiffness and hemodynamic measurements were performed using a SphygmoCor device (AtCor Medical, Sydney, Australia). An applanation tonometry was used for recording pressure waveforms that include cfPWV, aortic systolic/diastolic blood pressure, HR, AIx, AIx75, and SEVR. SEVR is calculated as area under the curve (AUC) during diastole/AUC during systole. In addition, baPWV and cfPWV were also evaluated with an oscillometric device (boso ABI-system 100; BOSCH \& SOHN, Germany).

\section{Heart Rate Variability (HRV)}

HRV was evaluated in both time and frequency domains using the SphygmoCor system (AtCor Medical, Sydney, Australia). For the time domain, the standard deviation of normal R-R intervals (SDNN), root mean square of successive $\mathrm{R}-\mathrm{R}$ interval differences (RMSSD), and the percentage of successive $\mathrm{R}-\mathrm{R}$ intervals that differed by $>50 \mathrm{~ms}$ (pNN50) were determined. SDNN reflects global HRV, whereas both RMSSD and pNN50 reflect parasympathetic activity. The analysis of the frequency spectrum was performed by applying a Fast Fourier Transform. Total power (TP) in the frequency range $(0-0.40 \mathrm{~Hz})$ was divided into two frequency bands: low frequency (LF: 0.04-0.15 $\mathrm{Hz}$, an index of sympathetic system modulation) and high frequency (HF: $0.15-0.40 \mathrm{~Hz}$, an index of parasympathetic system modulation). LF/HF is the ratio of LF to HF.

\section{Statistical Analysis}

All data analyses were performed using SPSS Version 16.0 (SPSS Inc., Chicago, IL, USA). A normality test was performed to assess data distribution, and variables that were not distributed normally were analyzed after log transformation. Paired-sample t-tests were used to evaluate the data pre- and post-intervention. Spearman correlation analysis was performed to measure the association between two variables. Data were reported as mean \pm SD. Cohen's d was used to calculate effect sizes on pairwise comparisons, with $0.2,0.5$, and 0.8 , representing small, medium, and large effects, respectively. ${ }^{9} P$-value $<0.05$ was considered statistically significant.

\section{Results}

\section{Effects Of Exercise And Diet Intervention On Anthropometric And Metabolic}

\section{Parameters}

As shown in Table 1, in response to the exercise and diet intervention, obese participants had significant reductions in body weight $(P=0.000, \mathrm{~d}=-2.91)$, BMI $(P=0.000$, $\mathrm{d}=-3.29)$, body fat mass $(P=0.000, \mathrm{~d}=-2.99)$, and body fat percentage $(P=0.000, \mathrm{~d}=-2.28)$. Obese subjects also had significantly lower concentrations of serum cholesterol $(P=0.000, \mathrm{~d}=-1.00)$ and LDL-c $(P=0.000, \mathrm{~d}=-1.35)$. Whereas, no significant changes in levels of fasting blood glucose, triglycerides $(P=0.063, \mathrm{~d}=-0.48)$, and HDL-c $(P=0.086, \mathrm{~d}=0.44)$ were found (Table 1$)$.

\section{Effects Of Exercise And Diet Intervention On PWV}

Using the boso ABI-system 100 device, we detected that baPWV on both left and right sides significantly reduced after intervention $(8.70 \pm 0.87 \mathrm{~m} / \mathrm{s}$ vs $7.69 \pm 0.93 \mathrm{~m} / \mathrm{s}$, $P=0.000, \mathrm{~d}=-1.45 ;$ and $8.47 \pm 0.91 \mathrm{~m} / \mathrm{s}$ vs $7.55 \pm 1.08$ $\mathrm{m} / \mathrm{s}, P=0.000, \mathrm{~d}=-1.35$; Table 2). Additionally, we found a reduction in cfPWV using both the boso device $(4.98 \pm 0.75 \mathrm{~m} / \mathrm{s}$ vs $4.22 \pm 0.88 \mathrm{~m} / \mathrm{s}, P=0.000$, $\mathrm{d}=-1.22)$ and SphygmoCor device $(5.32 \pm 0.85 \mathrm{~m} / \mathrm{s}$ vs $4.44 \pm 0.90 \mathrm{~m} / \mathrm{s}, P=0.000, \mathrm{~d}=-1.02$; Table 2). 
Table I The Anthropometric And Metabolic Parameters Of Subjects Before And After 6-Week Combined Exercise And Diet Intervention

\begin{tabular}{|l|l|l|l|}
\hline Parameters & Before & After & $P$ \\
\hline Height $(\mathrm{cm})$ & $162.4 \pm 9.04$ & $162.5 \pm 9.12$ & 0.654 \\
Body weight $(\mathrm{kg})$ & $80.9 \pm 16.8 \mathrm{I}$ & $70.4 \pm 14.06$ & 0.000 \\
BMI $\left(\mathrm{kg} / \mathrm{m}^{2}\right)$ & $30.4 \pm 3.7$ & $26.4 \pm 3.1$ & 0.000 \\
Body fat $(\mathrm{kg})$ & $33.2 \pm 8.5$ & $24.3 \pm 7.3$ & 0.000 \\
Body fat $(\%)$ & $40.9 \pm 4.7$ & $34.2 \pm 5.5$ & 0.000 \\
Cholesterol (mmol/L) & $4.18 \pm 0.84$ & $3.57 \pm 0.64$ & 0.000 \\
Triglycerides (mmol/L) & $0.71 \pm 0.30$ & $0.56 \pm 0.19$ & 0.063 \\
HDL-c (mmol/L) & $1.30 \pm 0.19$ & $1.39 \pm 0.18$ & 0.086 \\
LDL-c (mmol/L) & $2.19 \pm 0.61$ & $1.69 \pm 0.49$ & 0.000 \\
Blood glucose (mmol/L) & $4.38 \pm 0.49$ & $4.22 \pm 0.56$ & 0.382 \\
\hline
\end{tabular}

Note: Values are presented as mean \pm SD.

Abbreviations: BMI, body mass index; HDL-c, high-density lipoprotein cholesterol; LDL-c, low-density lipoprotein cholesterol.

Table 2 The Cardiovascular Function Parameters Of Subjects Before And After 6-Week Combined Exercise And Diet Intervention

\begin{tabular}{|l|l|l|l|}
\hline Parameters & Before & After & $P$ \\
\hline${\text { Left baPWV }(\mathrm{m} / \mathrm{s})^{\mathrm{a}}}_{\text {Right baPWV }(\mathrm{m} / \mathrm{s})^{\mathrm{a}}}$ & $8.70 \pm 0.87$ & $7.69 \pm 0.93$ & 0.000 \\
cfPWV (m/s) & $8.47 \pm 0.91$ & $7.55 \pm 1.08$ & 0.000 \\
cfPWV (m/s) & $4.98 \pm 0.75$ & $4.22 \pm 0.88$ & 0.000 \\
Augmentation pressure & $5.32 \pm 0.85$ & $4.44 \pm 0.90$ & 0.000 \\
(mmHg) $^{\mathrm{b}}$ & & $0.95 \pm 2.25$ & 0.261 \\
Alx75 $(\%)^{\mathrm{b}}$ & $5.90 \pm 14.64$ & $-4.95 \pm 7.46$ & \\
SEVR $(\%)^{\mathrm{b}}$ & $136.8 \pm 25.13$ & $173.7 \pm 32.45$ & 0.002 \\
HR (bPm) & $72.6 \pm 7.88$ & $61.4 \pm 6.42$ & 0.000 \\
bSBP $(\mathrm{mmHg})$ & $119.1 \pm 11.2$ & $108.6 \pm 8.5$ & 0.000 \\
bDBP $(\mathrm{mmHg})$ & $75.5 \pm 8.6$ & $65.3 \pm 6.1$ & 0.000 \\
aSBP $(\mathrm{mmHg})^{\mathrm{b}}$ & $103.7 \pm 8.58$ & $92.7 \pm 6.84$ & 0.000 \\
aDBP $(\mathrm{mmHg})^{\mathrm{b}}$ & $76.6 \pm 8.32$ & $65.7 \pm 5.97$ & 0.000 \\
\hline
\end{tabular}

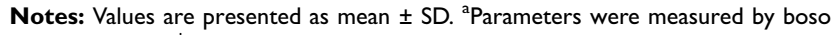
ABI-system 100. ' Parameters were measured by SphygmoCor device.

Abbreviations: baPWV, brachial-ankle pulse wave velocity; cfPWV, carotidfemoral pulse wave velocity; Alx75, augmentation index standardized to a heart rate of $75 / \mathrm{min}$; SEVR, subendocardial viability ratio; HR, heart rate; bSBP, brachial systolic blood pressure; bDBP, brachial diastolic blood pressure; aSBP, aortic systolic blood pressure; aDBP, aortic diastolic blood pressure.

\section{Effects Of Exercise And Diet Intervention On Central Hemodynamics}

Pulse wave reflection was evaluated by using SphygmoCor device. As shown in Table 2, obese adolescents showed significant reductions in AIx75 $(P=0.002, \mathrm{~d}=-0.80)$, resting heart rate $(P=0.000, \mathrm{~d}=-1.47)$, brachial SBP $(P=0.000, \mathrm{~d}=-1.40)$, brachial DBP $(P=0.000$, $\mathrm{d}=-1.58)$, aortic SBP $(P=0.000, \mathrm{~d}=-1.76)$, and aortic DBP $(P=0.000, \mathrm{~d}=-1.76)$ following exercise and diet intervention (Table 2). There was a significant augmentation in SEVR after the intervention $(P=0.000, \mathrm{~d}=0.92)$. However, there was no change in augmentation pressure (Table 2).

\section{Effects Of Exercise And Diet Intervention On Cardiac Autonomic Function}

When focusing on the HRV indices of obese individuals at the baseline and at the end of 6 weeks of training program (Table 3), we found a significant augmentation in levels of SDNN $(65.2 \pm 18.98 \mathrm{~ms}$ vs $88.3 \pm 28.36 \mathrm{~ms}$, $P=0.013, \mathrm{~d}=0.73), \mathrm{RMSSD}(65.8 \pm 27.49 \mathrm{~ms}$ vs $100.5 \pm$ $37.68 \mathrm{~ms}, P=0.008, \mathrm{~d}=0.80)$, and $\mathrm{pNN} 50(37.8 \% \pm$ $17.05 \%$ vs $57.8 \% \pm 12.31 \%, P=0.008, \mathrm{~d}=0.84)$ using time domain assessment. Moreover, we detected an augmentation in level of HF $(P=0.072, \mathrm{~d}=0.54)$ and a reduction in level of $\mathrm{LF} / \mathrm{HF}(P=0.047, \mathrm{~d}=-0.59)$ using frequency domain analysis. However, there were no changes in TP and LF (Table 3).

\section{Associations Of Changes In HRV Indices With Changes In Central Hemodynamics And Heart Rate After Exercise And Diet Intervention}

As shown in Table 4, correlation analysis showed that the change in SEVR was positively correlated with the changes in SDNN ( $\mathrm{r}=0.518, P=0.048)$, RMSSD $(\mathrm{r}=0.499, P=0.058), \mathrm{TP}(\mathrm{r}=0.518, P=0.084), \mathrm{LF}$ $(\mathrm{r}=0.520, P=0.047)$, and HF $(\mathrm{r}=0.459, P=0.085)$. Furthermore, the change in AIx75 was negatively correlated with the changes in TP $(\mathrm{r}=-0.655, P=0.021)$ and LF ( $\mathrm{r}=-0.673, P=0.006$ ). In addition, the change in resting heart rate was negatively correlated with

Table 3 The Heart Rate Variability Indices Of Subjects Before And After 6-Week Combined Exercise And Diet Intervention

\begin{tabular}{|l|l|l|l|}
\hline Parameters & Before & After & $\boldsymbol{P}$ \\
\hline SDNN (ms) & $65.2 \pm 18.98$ & $88.3 \pm 28.36$ & 0.013 \\
RMSSD (ms) & $65.8 \pm 27.49$ & $100.5 \pm 37.68$ & 0.008 \\
PNN50 (\%) & $37.8 \pm 17.05$ & $57.8 \pm 12.31$ & 0.008 \\
TP $\left(\mathrm{ms}^{2}\right)$ & $4012.0 \pm 2272.3$ & $4633.7 \pm 1978.1$ & 0.465 \\
LF $\left(\mathrm{ms}^{2}\right)$ & $1172.8 \pm 1189.5$ & $1882.8 \pm 2465.1$ & 0.371 \\
HF $\left(\mathrm{ms}^{2}\right)$ & $1372.5 \pm 908.8$ & $1988.0 \pm 983.3$ & 0.072 \\
LF/HF & $1.0 \pm 0.55$ & $0.7 \pm 0.43$ & 0.047 \\
\hline
\end{tabular}

Note: Values are presented as mean \pm SD.

Abbreviations: SDNN, standard deviation of normal R-R intervals; RMSSD, root mean square of successive R-R interval differences; $p N N 50$, percentage of successive $R-R$ intervals that differed by $>50 \mathrm{~ms}$; TP, total power; LF, low-frequency power; HF, high-frequency power; LF/HF, low-frequency power/high-frequency power. 
Table 4 Associations Of Changes In Heart Rate Variability Indices With Changes In Central Hemodynamics And Heart Rate After 6-Week Combined Exercise And Diet Intervention

\begin{tabular}{|l|l|l|l|l|l|l|}
\hline \multirow{2}{*}{} & \multicolumn{2}{l}{ SEVR } & \multicolumn{2}{l|}{ Alx75 } & \multicolumn{2}{l|}{ HR } \\
\cline { 2 - 7 } & $\mathbf{r}$ & $\boldsymbol{P}$ & $\mathbf{r}$ & $\boldsymbol{P}$ & $\mathbf{r}$ & $\boldsymbol{P}$ \\
\hline SDNN & 0.518 & 0.048 & -0.399 & 0.140 & -0.736 & 0.002 \\
RMSSD & 0.499 & 0.058 & -0.335 & 0.223 & -0.813 & 0.000 \\
PNN50 & 0.282 & 0.329 & -0.324 & 0.259 & -0.749 & 0.002 \\
TP & 0.518 & 0.084 & -0.655 & 0.021 & -0.172 & 0.593 \\
LF & 0.520 & 0.047 & -0.673 & 0.006 & -0.270 & 0.330 \\
HF & 0.459 & 0.085 & -0.290 & 0.294 & -0.748 & 0.001 \\
LF/HF & -0.04 & 0.893 & -0.249 & 0.391 & -0.485 & 0.079 \\
\hline
\end{tabular}

Abbreviations: SDNN, standard deviation of normal R-R intervals; RMSSD, root mean square of successive R-R interval differences; $P N N 50$, percentage of successive R-R intervals that differed by $>50 \mathrm{~ms}$; TP, total power; LF, low-frequency power; HF, high-frequency power; LF/HF, the ratio of LF to HF; SEVR, subendocardial viability ratio; Alx 75 , augmentation index standardized to a heart rate of 75 min; HR, heart rate.

the changes in SDNN ( $\mathrm{r}=-0.736, P=0.002)$, RMSSD $(\mathrm{r}=-0.813, P=0.000), \mathrm{pNN} 50(\mathrm{r}=-0.749, P=0.002)$, and $\mathrm{HF}(\mathrm{r}=-0.748, P=0.001)$.

\section{Discussion}

The results of the present study reported that exercise training and dietary restriction lead to improved anthropometrics, lipid metabolism, arterial function, and cardiac autonomic control of participants with obesity. To the best of our knowledge, this is the first study to assess the effects of a combined exercise and dietary intervention on arterial stiffness, central hemodynamics and autonomic function in obese adolescents. The results of this study revealed that obese adolescents had significantly lower central and systemic arterial stiffness, AIx 75, HR, brachial and aortic blood pressure, and they also had significantly higher SEVR and HRV after a 6-week exercise training and dietary restriction.

Overweight and obesity are important clinical and public health burdens worldwide. Obesity and cardiovascular disease are inextricably linked. Moreover, childhood and adolescent obesity are predisposed to an increased risk of cardiovascular disease in adulthood. Therefore, the importance and need for early detection and prevention of cardiovascular abnormalities to attenuate potentially progressive cardiovascular damage in obese children and adolescents have been highlighted. ${ }^{3}$

It has been reported that diet and exercise training program improved vascular function in obese children and adolescents. ${ }^{18,19}$ Likewise, our results showed that both cfPWV and baPWV were significantly reduced after 6-week intervention in obese adolescents, suggesting that both aortic and systemic arterial stiffness might be improved by changes in lifestyle. Since impaired arterial stiffness is the early diagnosis of atherosclerosis, our results indicate that 6 -week exercise and diet intervention are beneficial to improve the arterial stiffness.

SEVR has been considered as a surrogate measure of microvascular myocardial perfusion, ${ }^{10}$ and lower values of SEVR indicate poorer perfusion of the subendocardium. ${ }^{11}$ Previous studies have shown lower values of SEVR in patients with high cardiovascular risk. ${ }^{12,13}$ However, it has not yet been investigated whether SEVR is altered under lifestyle modifications of exercise and diet. The present study reported for the first time that SEVR was significantly enhanced in obese adolescents after the exercise and diet intervention. Our results suggest that a 6week exercise training and dietary restriction are effective in improving microvascular coronary perfusion in obese adolescents.

HRV has been considered as an index of cardiac autonomic function. Reduced HRV provided an early and sensitive indicator of cardiac impairment. Since the autonomic nervous system is an important determinant of regulating the cardiovascular system and energy expenditure, ${ }^{14}$ it is hypothesized to contribute to the pathophysiology of obesity. SDNN was used to represent the global HRV, ${ }^{15}$ and we found a significant increase in SDNN in obese adolescents after the exercise and diet intervention. Moreover, we had also observed that both RMSSD and pNN50 values were also enhanced after the intervention. Additionally, frequency domain analysis of HRV showed an increased level of HF and a decreased level of LF/HF after the intervention. These results indicated increased parasympathetic autonomic function. ${ }^{15}$ Consistent with the present study, a previous study has also reported that a weight reduction program induced a change in autonomic activity in children with obesity toward parasympathetic dominance. ${ }^{16}$

Cardiac autonomic nervous system plays a vital role in the regulation of heart rate and vascular tone. ${ }^{17}$ Thus, deterioration of autonomic nervous function may contribute to greater arterial stiffness in patients with obesity, ${ }^{18}$ type 1 diabetes mellitus ${ }^{17,19,20}$ and systemic sclerosis. ${ }^{21} \mathrm{~A}$ novel finding of our study was that an increased parasympathetic autonomic function was correlated with an improvement in central hemodynamics (i.e. increased SEVR and decreased AIx75) after an exercise and diet 
intervention. To the best of our knowledge, this is the first study to report a significant association between enhanced autonomic function and improved hemodynamics among obese adolescents undergoing lifestyle modifications of physical exercise and diet intervention. In accordance with our results, a previous study showed that 6 weeks of whole-body vibration training were effective at increasing autonomic function, associated with an augmentation in arterial function in young overweight and obese women. ${ }^{18}$ Taken together, these results suggest that the improvement in arterial function would be partially attributed to an increase in autonomic function after exercise and diet intervention.

Previous studies have reported that exercise or dietary modification may increase cardiac autonomic function ${ }^{22,23}$ and reduce arterial stiffness ${ }^{24,25}$ in obese subjects. The beneficial effects of exercise on autonomic function including vagal influence on the heart and arterial baroreflex sensitivity have been reported. ${ }^{26}$ The arterial baroreflex plays an important role in modulating heart rate and blood vessel tone to maintain arterial blood pressure. Indeed, an increase in arterial baroreflex sensitivity with an associated reduction in arterial stiffness has been observed following exercise. ${ }^{26}$ Furthermore, a significant negative correlation between augmented HRV and decreased resting heart rate was observed in the present study, indicating that the effects of a 6-week program of exercise and dietary restriction on cardiac autonomic modulation and arterial function were partly due to the changes in resting heart rate as well as changes in systolic and diastolic blood pressure. Future studies are needed to examine the mechanisms underlying the association between changes in cardiac autonomic function and arterial function after life modifications.

The present study sought to determine the beneficial effects of a 6-week exercise training with dietary restriction on cardiac and vascular function as well as body composition and lipid metabolism in obese adolescents. Herein, our results revealed that the exercise and diet intervention resulted in significant augmentations in central hemodynamics and autonomic function with a reduction in arterial stiffness. Furthermore, this intervention proved to effectively decrease body weight, BMI and body fat with improvements in lipid metabolism. In agreement with our findings, previous studies observed reductions in body weight and body fat after 4 or 10 weeks ${ }^{27,28}$ of exercise and diet intervention in obese children and adolescents. Taken together, these results suggest that our program of combined exercise and dietary restriction is an effective intervention in the improvement of body composition as well as metabolic milieus, thereby preventing the development of cardiac and vascular dysfunction in obese adolescents.

There are several limitations to our current study. For example, a control group was lacked during the training program of the intervention. Because the camp program was a traditional residential camp with a specific aim of weight loss. The camp was located in a remote district of the city, and all obese subjects came from all over the country. Therefore, we thought it difficult to recruit an appropriate control group, which had been previously described. ${ }^{8}$ In addition, the number of participants was relatively small. Future research on a larger sample size will be conducted.

In conclusion, this study revealed that obese adolescents had improved central hemodynamics and autonomic function with reduced arterial stiffness after a 6-week exercise training and dietary restriction. Furthermore, a positive correlation between an enhancement of cardiac autonomic function and an improvement in central hemodynamics was found after the intervention.

\section{Data Sharing Statement}

The clinical study data used to support the findings of this study are available from the corresponding author upon request.

\section{Acknowledgments}

The present study was supported by the National Natural Science Foundation of China (grant numbers 31771315, 31600969) and the Natural Science Foundation of Guangdong Province (grant number 2016A030313625).

\section{Disclosure}

The authors report no conflicts of interest in this work.

\section{References}

1. Gungor NK. Overweight and obesity in children and adolescents. $J$ Clin Res Pediatr Endocrinol. 2014;6(3):129-143.

2. Gielen S, Schuler G, Adams V. Cardiovascular effects of exercise training: molecular mechanisms. Circulation. 2010;122(12):12211238. doi:10.1161/CIRCULATIONAHA.110.939959

3. Acree LS, Comp PC, Whitsett TL, et al. The influence of obesity on calf blood flow and vascular reactivity in older adults. Dyn Med. 2007;6:4. doi:10.1186/1476-5918-6-4

4. Van Guilder GP, Hoetzer GL, Dengel DR, Stauffer BL, DeSouza CA. Impaired endothelium-dependent vasodilation in normotensive and normoglycemic obese adult humans. $J$ Cardiovasc Pharmacol. 2006;47(2):310-313. doi:10.1097/01.fjc.0000205097.29946.d3 
5. Lee JH, Kang YE, Chang JY, et al. An engineered FGF21 variant, LY2405319, can prevent non-alcoholic steatohepatitis by enhancing hepatic mitochondrial function. Am J Transl Res. 2016;8(11):4750 4763.

6. Mifflin MD, St Jeor ST, Hill LA, Scott BJ, Daugherty SA, Koh YO. A new predictive equation for resting energy expenditure in healthy individuals. Am J Clin Nutr. 1990;51(2):241-247. doi:10.1093/ajcn/ 51.2.241

7. Weijs PJ. Validity of predictive equations for resting energy expenditure in US and Dutch overweight and obese class I and II adults aged 18-65 y. Am J Clin Nutr. 2008;88(4):959-970. doi:10.1093/ ajen/88.4.959

8. Huang J, Wang $\mathrm{S}, \mathrm{Xu} \mathrm{F}$, et al. Exercise training with dietary restriction enhances circulating irisin level associated with increasing endothelial progenitor cell number in obese adults: an intervention study. PeerJ. 2017;5:e3669. doi:10.7717/peerj.3669

9. Lakens D. Calculating and reporting effect sizes to facilitate cumulative science: a practical primer for t-tests and ANOVAs. Front Psychol. 2013;4:863. doi:10.3389/fpsyg.2013.00186

10. Turzyniecka M, Wild SH, Krentz AJ, Chipperfield AJ, Clough GF, Byrne CD. Diastolic function is strongly and independently associated with cardiorespiratory fitness in central obesity. $J$ Appl Physiol. 2010;108(6):1568-1574. doi:10.1152/japplphysiol.00023.2010

11. Doonan RJ, Scheffler P, Yu A, et al. Altered arterial stiffness and subendocardial viability ratio in young healthy light smokers after acute exercise. PLoS One. 2011;6(10):e26151. doi:10.1371/journal. pone.0026151

12. Di Micco L, Salvi P, Bellasi A, Sirico ML, Di Iorio B. Subendocardial viability ratio predicts cardiovascular mortality in chronic kidney disease patients. Blood Purif. 2013;36(1):26-28. doi:10.1159/000350582

13. Laugesen E, Hoyem P, Fleischer J, et al. Reduced subendocardial viability ratio is associated with unfavorable cardiovascular risk profile in women with short duration of type 2 diabetes. $A m J$ Hypertens. 2016;29(10):1165-1172. doi:10.1093/ajh/hpw066

14. Mathieu P, Pibarot P, Larose E, Poirier P, Marette A, Despres JP. Visceral obesity and the heart. Int J Biochem Cell Biol. 2008;40 (5):821-836. doi:10.1016/j.biocel.2007.12.001

15. Salamin G, Pelletier C, Poirier P, et al. Impact of visceral obesity on cardiac parasympathetic activity in type 2 diabetics after coronary artery bypass graft surgery. Obesity. 2013;21(8):1578-1585. doi:10.1002/oby.20089

16. Mazurak N, Sauer H, Weimer K, et al. Effect of a weight reduction program on baseline and stress-induced heart rate variability in children with obesity. Obesity. 2016;24(2):439-445. doi:10.1002/oby.21355
17. Jaiswal M, Urbina EM, Wadwa RP, et al. Reduced heart rate variability is associated with increased arterial stiffness in youth with type 1 diabetes: the SEARCH CVD study. Diabetes Care. 2013;36 (8):2351-2358. doi:10.2337/dc12-0923

18. Figueroa A, Gil R, Wong A, et al. Whole-body vibration training reduces arterial stiffness, blood pressure and sympathovagal balance in young overweight/obese women. Hypertens Res. 2012;35(6):667672. doi: $10.1038 / \mathrm{hr} .2012 .15$

19. Liatis S, Alexiadou K, Tsiakou A, Makrilakis K, Katsilambros N, Tentolouris N. Cardiac autonomic function correlates with arterial stiffness in the early stage of type 1 diabetes. Exp Diabetes Res. 2011;2011:957901. doi:10.1155/2011/957901

20. van Ittersum FJ, Schram MT, van der Heijden-Spek JJ, et al. Autonomic nervous function, arterial stiffness and blood pressure in patients with type I diabetes mellitus and normal urinary albumin excretion. J Hum Hypertens. 2004;18(11):761-768. doi:10.1038/sj.jhh.1001751

21. Gigante A, Rosato E, Liberatori M, et al. Autonomic dysfunction in patients with systemic sclerosis: correlation with intrarenal arterial stiffness. Int $J$ Cardiol. 2014;177(2):578-580. doi:10.1016/j. ijcard.2014.08.122

22. Felber Dietrich D, Ackermann-Liebrich U, Schindler C, et al. Effect of physical activity on heart rate variability in normal weight, overweight and obese subjects: results from the SAPALDIA study. Eur $J$ Appl Physiol. 2008;104(3):557-565. doi:10.1007/s00421-008-0800-0

23. Lind L, Andren B. Heart rate recovery after exercise is related to the insulin resistance syndrome and heart rate variability in elderly men. Am Heart J. 2002;144(4):666-672. doi:10.1067/mhj.2002.124836

24. Goldberg Y, Boaz M, Matas Z, Goldberg I, Shargorodsky M. Weight loss induced by nutritional and exercise intervention decreases arterial stiffness in obese subjects. Clin Nutr. 2009;28(1):21-25. doi:10.1016/j.clnu.2008.10.001

25. Maeda S, Zempo-Miyaki A, Sasai H, Tsujimoto T, So R, Tanaka K. Lifestyle modification decreases arterial stiffness in overweight and obese men: dietary modification vs. exercise training. Int J Sport Nutr Exerc Metab. 2015;25(1):69-77. doi:10.1123/ijsnem.2013-0107

26. Heydari M, Boutcher YN, Boutcher SH. High-intensity intermittent exercise and cardiovascular and autonomic function. Clin Auton Res. 2013;23(1):57-65. doi:10.1007/s10286-012-0179-1

27. Gronbaek H, Lange A, Birkebaek NH, et al. Effect of a 10-week weight loss camp on fatty liver disease and insulin sensitivity in obese Danish children. J Pediatr Gastroenterol Nutr. 2012;54 (2):223-228. doi:10.1097/MPG.0b013e31822cdedf

28. Wang R, Chen PJ, Chen WH. Effect of diet and exercise-induced weight reduction on complement regulatory proteins $\mathrm{Cd} 55$ and $\mathrm{Cd} 59$ levels in overweight chinese adolescents. J Exerc Sci Fit. 2011;9 (1):46-51. doi:10.1016/S1728-869X(11)60006-3

Diabetes, Metabolic Syndrome and Obesity: Targets and Therapy

Dovepress

Publish your work in this journal

Diabetes, Metabolic Syndrome and Obesity: Targets and Therapy is an international, peer-reviewed open-access journal committed to the rapid publication of the latest laboratory and clinical findings in the fields of diabetes, metabolic syndrome and obesity research. Original research, review, case reports, hypothesis formation, expert opinion and commentaries are all considered for publication. The manuscript management system is completely online and includes a very quick and fair peer-review system, which is all easy to use. Visit http://www.dovepress.com/testimonials.php to read real quotes from published authors. 